Article

\title{
An Intelligent Wireless System for Field Ecology Monitoring and Forest Fire Warning
}

\author{
Yili Zheng *, Yandong Zhao, Weiping Liu, Shengbo Liu and Ruting Yao
}

School of Technology, Beijing Forestry University, Beijing 100083, China; yandongzh@bjfu.edu.cn (Y.Z.); hfp1916@126.com (W.L.); podolski@bjfu.edu.cn (S.L.); Yaoruting@bjfu.edu.cn (R.Y.)

* Correspondence: zhengyili@bjfu.edu.cn; Tel.: +86-10-6233-7736

Received: 20 November 2018; Accepted: 14 December 2018; Published: 16 December 2018

\begin{abstract}
Based on Internet-of-Things and multi-sensor technology, an intelligent wireless monitoring system was developed to obtain field ecological parameters and provide forest fire warning in real-time. The GPRS and China's Beidou satellite communication were selectively used for date transmission in the field with weak cell phone signals. This monitoring system is mainly composed of several field ecological monitoring stations, a cloud server, and online system software. Atmosphere, soil, sunlight and plant parameters of different regions are obtained real-time by sensors stably and reliably. This system has functions such as field ecological data storage, dynamic query, report generation, and data analysis. As an example of typical application, the forest fire weather grade, which was supplemented with the litter layer soil humidity, was calculated to realize the early warning of the local forest fire in this system through continuous experiments at Beijing Jiufeng National Forest Park from March to May 2017 and Inner Mongolia from March to June 2018. The success ratios of data transmission through Beidou satellite were 98.57\%, 99.43\%, $99.59 \%$, and $98.85 \%$, respectively, in Beijing, and through GPRS were $99.89 \%$ and $99.90 \%$ in Inner Mongolia. Long-term real-time field ecological monitoring and forest fire warning were successfully realized. This system can be widely used for big data field acquisition and analysis in forest and agriculture regions.
\end{abstract}

Keywords: Internet-of-Things; intelligent wireless monitoring; Beidou satellite; forest fire warning

\section{Introduction}

The real-time continuous monitoring of field ecological system parameters can generate massive big data information sources to reveal the relationships between various ecological factors and their internal variation rules, support ecological conservation actions, and prevent ecological disasters [1].

Currently, the methods for obtaining the parameters for monitoring field ecology, such as atmosphere, soil, light, and plants, fall into four classes. Class 1 consists in consulting the official website of the National Meteorological Observatory for meteorological data. However, the terrain, altitude, and vegetation conditions in a forestry vary substantially, and the unique microclimate environment significantly deviates from the data on the website for the National Meteorological Observatory, thus making it impossible to guarantee the timeliness and accuracy of monitoring data [2-4]. Class 2 consists in monitoring the meteorological data or spectroscopic data in a forest region using a meteorological satellite or remote sensing satellite [5-7]. This method can only achieve macroscopic wide-range monitoring and early warning of the ecological changes and disasters in forests and cannot obtain fine microscopic data. Class 3 consists in deploying a large number of wireless sensor nodes in a field region [8-13]. Sensor nodes can collect microclimate data and send them to their respective cluster nodes by wireless sensor networks. The wireless sensor networks in the field face obstacles such as limited power resources and a high vulnerability to harsh environment. 
Class 4 consists in building designated ecological monitoring stations in the forest region [14-16] to automatically measure the ecological microenvironment data of farmland and forests. This method is widely used because of its advantages, such as high accuracy, saving of labor, and capacity to continuously monitor for a long time. Many ground-based ecological monitoring stations are built to collect the visual or infrared images and microclimate data for forest fire warnings [17-19]. Based on static and dynamic analysis of chromatic changes, histogram changes, or luminance changes in images, forest fires can be detected and classified. Different sensor data are used to reduce or eliminate the false alarm rate. In some remote regions or regions without mobile phone signal, this method cannot achieve an effective real-time transmission of data and images, which becomes a bottleneck for its actual application in monitoring field ecology.

The Beidou Satellite, developed by China, is a global satellite navigation system capable of providing positioning and navigation, two-way short message communication, and precise timing services at any location and at any time within the coverage area, thus effectively solving the problem of data communication in remote regions and regions with no or weak mobile phone signal [20-22].

Based on the communication of China's Beidou Satellite and on GPRS communication, this paper develops a real-time monitoring network system for field ecological parameters and achieves real-time monitoring of various parameters such as atmosphere, soil, light, and plants of the forest as well as remote transmission, cloud storage, and query of data. The system serves the acquisition and analysis of ecological big data in remote regions and regions with strong or weak mobile phone signal.

\section{Design of the Monitoring System}

\subsection{System Composition}

The system comprises multiple field ecological monitoring stations, a cloud server, and online system software, as shown in Figure 1.

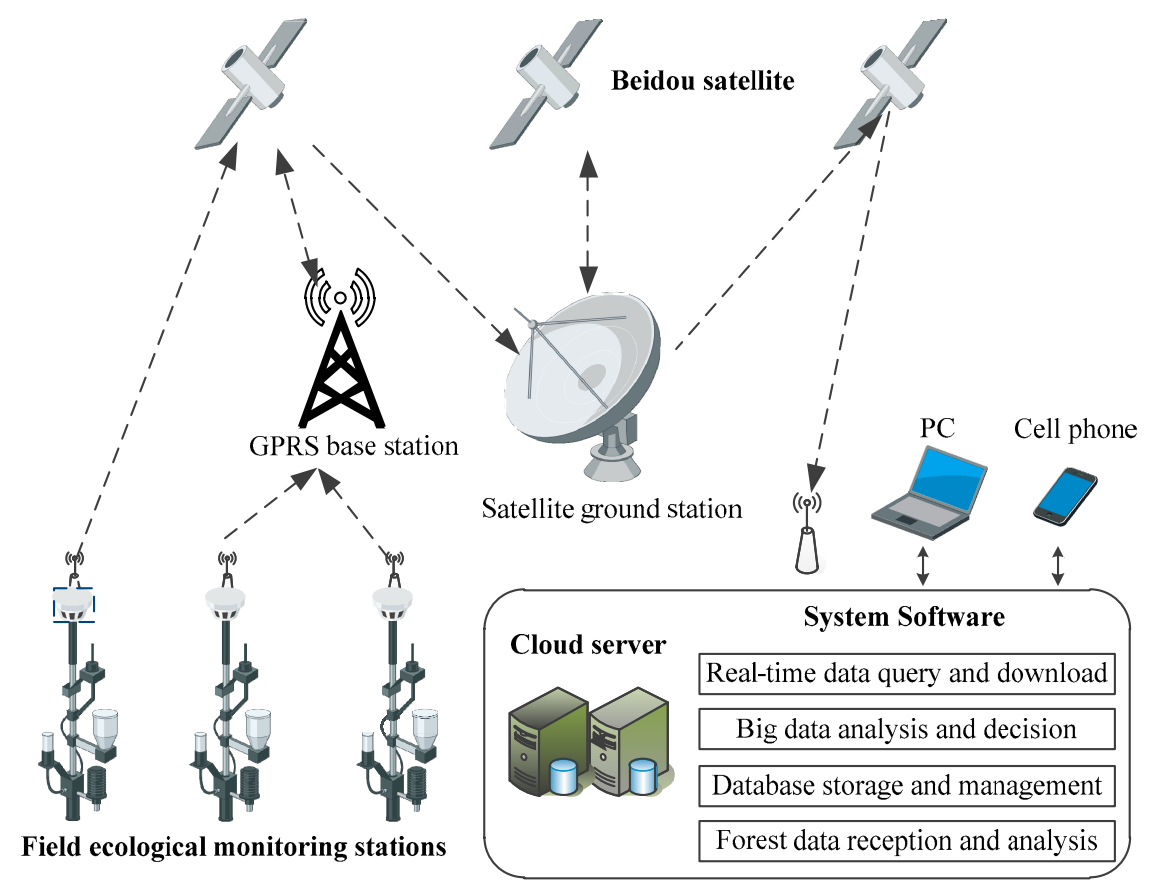

Figure 1. Schematic diagram of the field monitoring system.

The field ecological monitoring station is connected with the sensor groups of atmosphere, soil, light, and plant parameters and is driven by solar energy. Two-way communication occurs between the field ecological monitoring station and the cloud server via the short message communication module of the Beidou satellite or GPRS. The system software runs on the cloud 
server provided by the Alibaba Cloud with functions such as real-time data query and download, big data analysis and decision-making, database storage and management, satellite data reception, and parsing. Multiple field ecological monitoring stations are networked for all-round monitoring of the microenvironment in forest regions. Users may inquire and download the ecology monitoring data of forest regions using a PC or a mobile phone via remote network connection.

The field ecological monitoring stations automatically acquire data from the connected sensors for atmosphere, soil, light, and plants at an interval of $10 \mathrm{~min}$, manually adjustable, according to their own clocks. In the forest region with weak mobile phone signal, the data are stored and reformatted to form a 72-byte short message communication package and sent to the Beidou Satellite through the communication module of the Beidou Satellite. The ground station of the Beidou Satellite receives the satellite data and in return sends the data to the cloud server through the Beidou Satellite. In the forest region with a stable mobile phone signal, GPRS is used for data transmission.

The system software can receive and parse the communication package of the Beidou satellite or GPRS, and then store the monitoring data into the MySQL database relying on its functions of receiving and parsing the satellite data. The real-time data query and download function allows for real-time access to the database. PC and mobile phone users can enjoy the services, such as remote data access, curve query, and statement download, through the Internet or the mobile communication network. The database storage and management function allows for operations of the data in the MySQL database, such as addition, deletion, modification, updating, and search.

The big data analysis and decision-making function of the system software allows for a determination of the grade of fire risk weather in forest regions, prediction and early warning of fire disasters in forests, an evaluation of the ecological environment restoration, decision-making on accurate irrigation, monitoring of growth state of stumpage, etc., according to the microclimate monitoring data acquired and the national standards or big data analysis models.

\subsection{Design of Online System Hardware}

The hardware of the field ecological monitoring station in forest regions is comprised of data acquisition units, a power supply system, sensor groups, Beidou Satellite communication modules, GPRS communication modules, and mechanical towers, as shown in Figure 2.

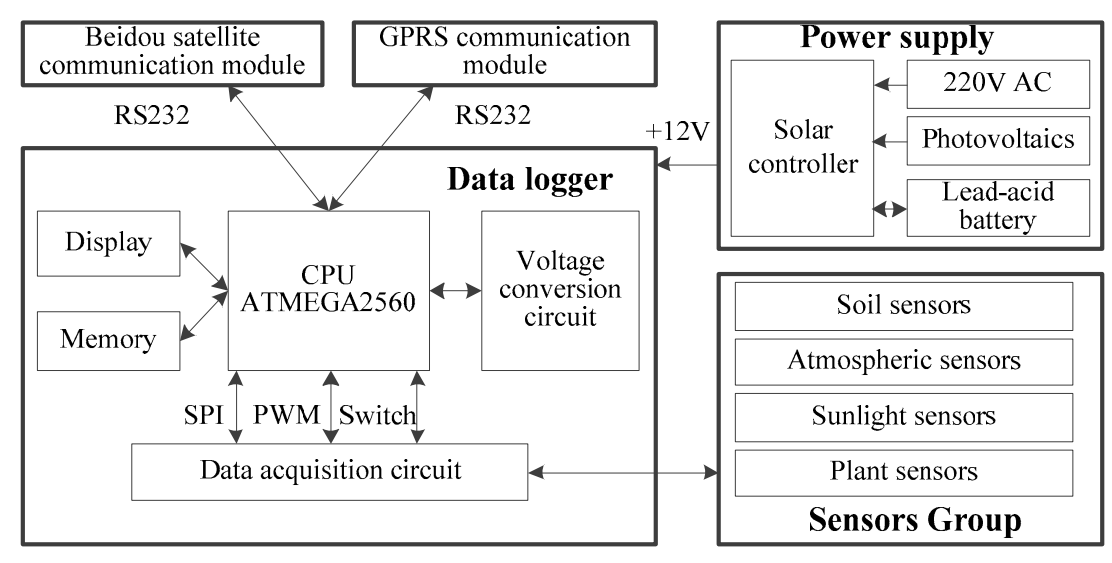

Figure 2. Block diagram of the system hardware.

The data acquisition unit is the data processing and control core of the whole field ecological monitoring station, composed of a central processing unit, a display circuit, a storage circuit, a data acquisition circuit, etc. The data acquisition unit can acquire the analog or digital signal output by each sensor and complete data transmission through the Beidou Satellite as required by users.

The central processing unit of the data acquisition unit uses the ATMEGA2560 single chip microcomputer of the ATMEL Company. The central processing unit completes the parsing of the original data from each sensor, the conversion of the data communication format, the conversion 
of the data display format, the conversion of the data storage format, the monitoring of the sensor operation mode, the monitoring of the satellite and GPRS communication mode, and the monitoring of the power supply mode and achieves automatic reset following operator errors via the watchdog circuit. A color monitor is used for displaying the monitoring data and status data. The data storage circuit uses an SD card with a storage capacity of $8 \mathrm{G}$ for locally storing monitoring data, allowing for manual data copying and erasing at regular intervals. The data acquisition circuit uniformly converts a 0-20 mA analog current signal and a $0-5 \mathrm{~V}$ analog voltage signal output, using the sensor group, into a $0-2.5 \mathrm{~V}$ analog voltage signal and is connected to the $\mathrm{AD}$ conversion interface of the single chip microcomputer after amplification and filtering. The IO interface of ATMEGA2560 acquires the switching value output via the precipitation sensor. The PWM interface acquires the output of the wind speed sensor.

The communication module of the Beidou Satellite uses the GYT2015C Beidou Satellite data transmission module. The data transmission module has the Beidou Satellite communication and Beidou Satellite positioning and timing functions and is connected to the central processing unit via the RS232 interface, with a transmitting power of $10 \mathrm{~W}$. The communication module of the Beidou Satellite is installed on the mechanical rack for providing the communication link of the Beidou Satellite for the field ecological monitoring station. The system reserves a GPRS communication module interface for use in the regions with mobile phone signal.

The sensor group can measure the parameters of soil, atmosphere, light, and plants in the ecological microenvironment in forest regions in real time. The major sensor configuration and properties are shown in Table 1. Users may increase or decrease the number of sensors as necessary.

Table 1. Sensor configuration and performance of the forest microclimate monitoring station.

\begin{tabular}{ccc}
\hline Group & Sensor Type & Range \\
\hline \multirow{2}{*}{ soil parameters } & soil temperature (2-6 layers) & $-55-+80^{\circ} \mathrm{C}$ \\
& soil moisture (2-6 layers) & $0-100 \%$ \\
\hline & air temperature & $-55-+80^{\circ} \mathrm{C}$ \\
& air moisture & $0-100 \%$ \\
air parameters & carbon dioxide concentration & $400-5000$ parts per million \\
& wind direction & $0-360^{\circ}$ \\
& wind speed & $0.3-60 \mathrm{~m} / \mathrm{s}$ \\
& precipitation & $0-4 \mathrm{~mm} / \mathrm{min}$ \\
& negative oxygen ion & $0-50,000 \mathrm{Ions} / \mathrm{cm}^{3}$ \\
light parameters & global radiation & $0-500 \mu \mathrm{g} / \mathrm{m}^{3}$ \\
\hline \multirow{2}{*}{ plant parameters } & light intensity & $0-2000 \mathrm{~W} / \mathrm{m}^{2}$ \\
& sunshine hours & $0-200,000 \mathrm{Lux}$ \\
& photosynthetically active radiation & threshold $120 \mathrm{~W} / \mathrm{m}^{2}$ \\
& needle-type stem moisture & $0-4000 \mu \mathrm{mol} / \mathrm{m}^{2} / \mathrm{s}$ \\
\hline
\end{tabular}

The power supply system is comprised of a solar energy and controller, a 75 Watts polycrystalline solar panel, and a $12 \mathrm{~V} 65 \mathrm{~A} \cdot \mathrm{h}$ gel energy storage battery and provides a $12 \mathrm{~V}$ direct current for the field ecological monitoring stations. A $220 \mathrm{~V}$ alternating current power supply can be used for the forest where mains supply is available. The energy storage battery is placed in an underground water-proof box and buried $0.5 \mathrm{~m}$ below the soil surface under the mechanical tower for anti-freezing protection.

The mechanical tower is made of stainless steel, fixed with stainless steel wire ropes for wind protection, and provided with a lightning conductor, with its foundation fixed by heavy concrete blocks. The mechanical tower is equipped with atmosphere parameter sensor groups, light parameter sensor groups, Beidou satellite and GPRS data transmission modules, and solar energy panels at the top. The mechanical tower is equipped with a stainless-steel rain-proof box in the middle and other 
components inside it, such as data acquisition unit and a solar energy controller. The soil parameter sensor group includes multiple soil moisture and temperature sensors, which may be buried in the soil at different depths in different positions for measuring the temperatures and moistures of the soil in forests. In terms of plant parameters, a non-destructive packaged stumpage stem moisture sensor and a needle-type stumpage stem moisture sensor are used to measure the volumetric moisture of stumpage stems with a diameter at breast height smaller than $10 \mathrm{~cm}$ and a diameter at breast height greater than $10 \mathrm{~cm}$, respectively.

Figure $3 a-d$ presents the three ecological monitoring stations using Beidou satellite communication located on Yan'er Ridge (at an altitude of $440 \mathrm{~m}$ ), Wangjing Tower (at an altitude of $750 \mathrm{~m}$ ), and Luobadi Peak (at an altitude of $1000 \mathrm{~m}$ ) in Beijing Jiufeng National Forest Park. No stable GPRS communication can be achieved for cell phones, as this region is far from the mobile base station and affected by the air route. Figure 4e-f present the two ecological monitoring stations using GPRS communication located on and Hulun Buir and Zhalantun in Inner Mongolia. No stable Beidou satellite short message communication can be achieved, as this region is far from the geosynchronous orbit of the Beidou satellite.

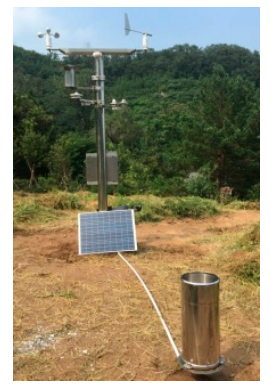

(a)

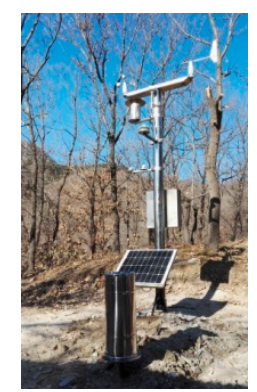

(d)

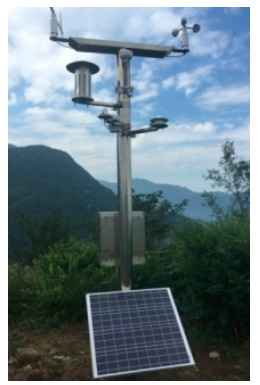

(b)

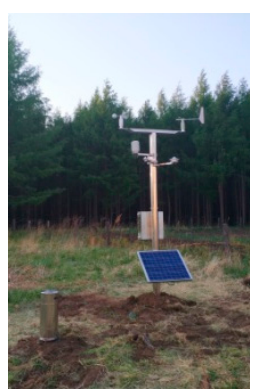

(e)

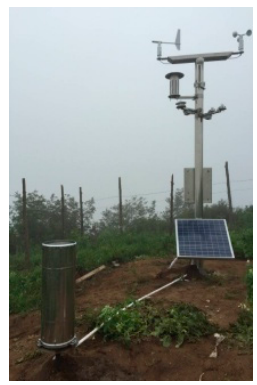

(c)

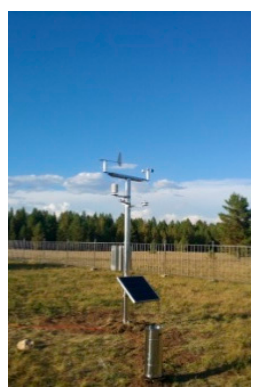

(f)

Figure 3. Forest microclimate monitoring stations in Beijing and Inner Mongolia. (a) Yan'er Ridge; (b) Wangjing Tower; (c) Luobadi; (d) Zhai'er Yu; (e) Hulun Buir; (f) Zhalantun.

\subsection{Design of System Software}

The system software runs continuously on the Alibaba cloud server. The overall framework is constructed based on the. NET technology, including the front-end presentation layer, the business logic layer, and the data access layer. Figure 4 presents the overall architecture of the system software.

The presentation layer is responsible for interactions between the system and multiple concurrent users. The business logic layer deals with the core businesses, such as monitoring data reception, big data analysis, and the logical processing of data to meet the requirements of the presentation layer and the monitoring tasks. The data access layer directly operates the database for addition, deletion, modification, updating, and search of the monitoring data. 


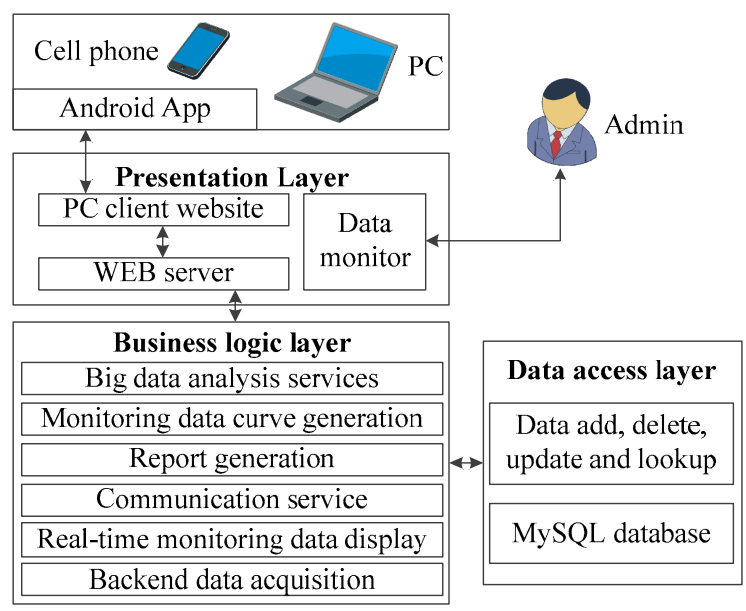

Figure 4. Block diagram of the software.

The PC front-end website of the presentation layer is designed with HTML, CSS, and the JavaScript scripting language. The visualized chart database Echarts.JS of Baidu is used to design the charts, dashboards, and dynamic components [23] for the monitoring data. In terms of data monitoring program, the Winform technique is used for setting up web pages. The administrators can monitor the operating state of the communication between the sensor and the Beidou Satellite. The WEB server program uses IIS of Microsoft and exchanges information with users' PC browsers via HTTP (hypertext transmission protocol).

The Java language is used to develop the Android application for mobile phones. The HTTP protocol is used to upload and download data to and from the cloud server. The MPAndroidChart open-source framework is used to achieve the query and display of real-time data on forest ecology, variation curve, grade of fire weather, etc.

The communication service program receives the monitoring data and operative mode data transmitted by the field ecological monitoring station via the short message communication of the Beidou Satellite or GPRS. The monitoring data generation program is used to generate daily statements, monthly statements, and annual statements of monitoring data in Excel format. The monitoring data curve program is used to generate daily variation, monthly variation, and annual variation curves for various ecological parameters. The real-time data generation program provides real-time varying data for the dashboard controls of the PC front-end website. The big data analysis service program can achieve evaluation of the ecological environment restoration, making decisions on accurate irrigation, the monitoring of the stumpage growth state, etc. The back-end data acquisition program is connected with the data monitoring program of the presentation layers to complete the parameter setting for the data acquisition unit, the parsing of data from various sensors, format conversion, and the automatic processing of errors and missing data.

The data access layer uses the MySQL database of open source code to provide storage space for all data and utilizes the structured query language SQL for database management.

The system software uses Load Runner for testing, supports 1200 concurrent users, and reserves interfaces for subsequent application and extension; it therefore meets requirements such as real-time capability, multiple concurrence, and extendibility.

Figures 5-7 present screenshots of the PC website. Figure 5 shows the real-time data on parameters such as temperature, moisture, and precipitation in the forest microenvironment in the form of a dashboard. Figure 6 shows the changes in forest temperature in one week in the form of a curve. Figure 7 shows ecological monitoring data of the forest microenvironment in the form of a statement. The data statements can be exported in Excel format. 


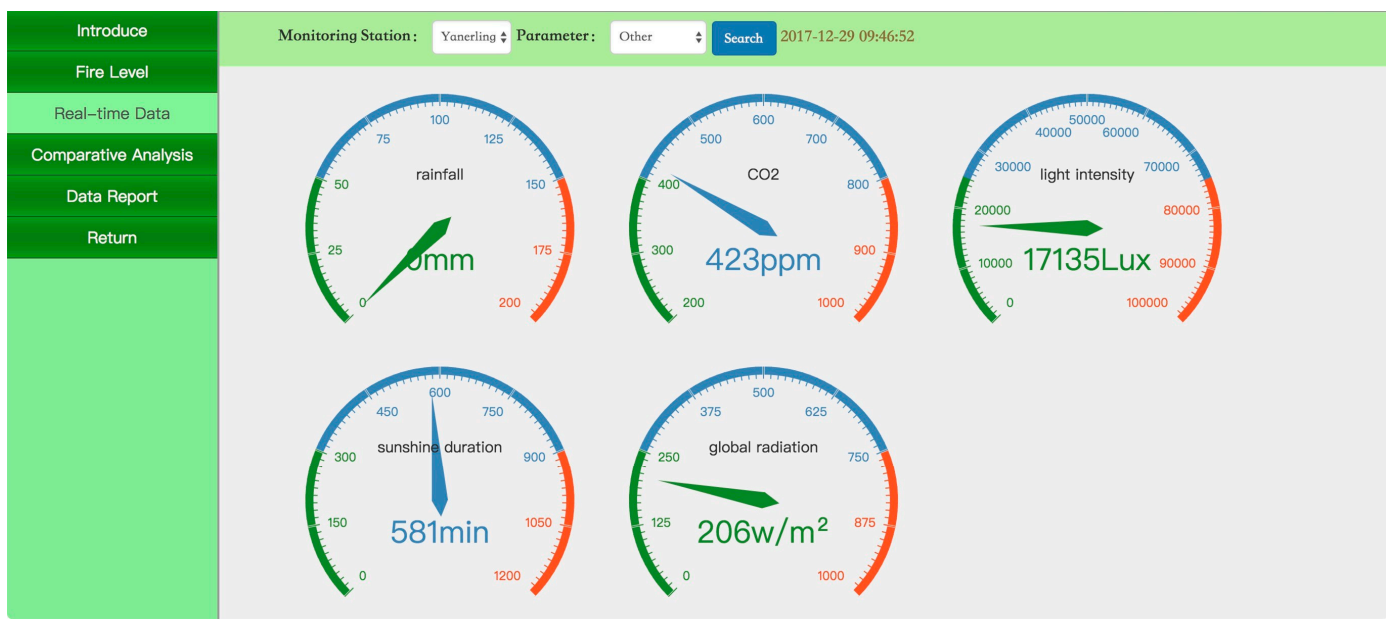

Figure 5. Real-time monitoring data of the forest microclimate.

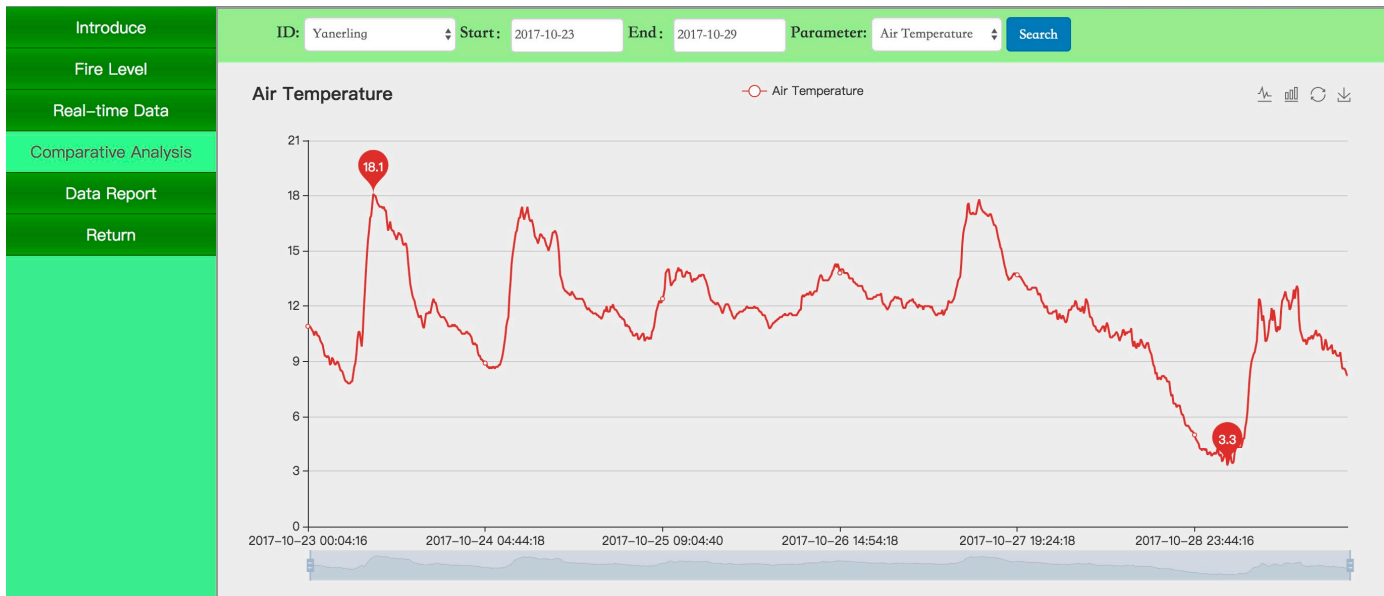

Figure 6. Air temperature curve of the forest microclimate.

\begin{tabular}{|c|c|c|c|c|c|c|c|c|c|}
\hline \multirow{2}{*}{\begin{tabular}{|c|} 
Introduce \\
Fire Level \\
\end{tabular}} & Monitoring Station: & iे Start: & 2017-10-23 & \multicolumn{2}{|c|}{ End: $2017-10-29$} & arch & & \multirow[b]{2}{*}{ ":! } & \multirow[b]{2}{*}{ A } \\
\hline & \multirow{2}{*}{ time } & \multirow[b]{2}{*}{ soil water in $1 . .}$. & \multirow[b]{2}{*}{ soil water in $2 \ldots$} & \multirow[b]{2}{*}{ soil temperat... } & \multirow[b]{2}{*}{ soil temperat... } & \multirow[b]{2}{*}{ wind speed(m/s) } & & & \\
\hline & & & & & & & \multicolumn{2}{|l|}{ wind direction(") } & air temper \\
\hline & $2017-10-2300: 04: 16$ & 32.4 & 0.2 & 11.8 & 12.9 & 0.3 & 359 & & 10.9 \\
\hline Data Report & $2017-10-2300: 14: 16$ & 32.4 & 0.2 & 11.8 & 12.9 & 0.3 & 359 & & 10.9 \\
\hline \multirow[t]{11}{*}{ Return } & 2017-10-23 00:24:15 & 32.4 & 0.2 & 11.8 & 12.9 & 0.3 & 352 & & 10.9 \\
\hline & $2017-10-23$ 00:34:16 & 32.4 & 0.2 & 11.8 & 12.9 & 0.5 & 352 & & 10.8 \\
\hline & $2017-10-23$ 00:44:17 & 32.4 & 0.2 & 11.8 & 12.9 & 0.4 & 359 & & 10.7 \\
\hline & $2017-10-2300: 54: 16$ & 32.4 & 0.2 & 11.8 & 12.9 & 0.4 & 359 & & 10.6 \\
\hline & 2017-10-23 01:04:16 & 32.4 & 0.2 & 11.8 & 12.9 & 0.3 & 299 & & 10.4 \\
\hline & $2017-10-2301: 14: 16$ & 32.4 & 0.2 & 11.8 & 12.9 & 0.7 & 186 & & 10.6 \\
\hline & 2017-10-23 01:24:16 & 32.4 & 0.2 & 11.8 & 12.9 & 0.6 & 202 & & 10.6 \\
\hline & $2017-10-23$ 01:34:16 & 32.4 & 0.2 & 11.8 & 12.9 & 0.4 & 201 & & 10.4 \\
\hline & $2017-10-2301: 44: 16$ & 32.4 & 0.2 & 11.8 & 12.9 & 0.4 & 205 & & 10.4 \\
\hline & 2017-10-23 01:54:16 & 32.4 & 0.2 & 11.8 & 12.9 & 0.3 & 359 & & 10.3 \\
\hline & $2017-10-23$ 02:04:16 & 32.4 & 0.2 & 11.8 & 12.9 & 0.4 & 359 & & 10.1 \\
\hline
\end{tabular}

Figure 7. Monitoring data reports of the forest microclimate.

\section{Prediction of the Fire Weather Grade}

The fire weather grade is an important index for measuring the possibility of forest fire occurrence and the difficulty level of its spread. Additionally, it is an important basis for forest fire protection management. The forecasts made by the Bureau of Meteorology are based on macroscopic data and 
are not specific enough to predict the grade of forest fire risk under different altitude, vegetation, or microclimate conditions. The microenvironment monitoring system for forest ecology can acquire and compute the local meteorological factors required for forecasting the grade of forest fire risk in real time, which plays an important role in improving the fire weather forecast and correct decision making.

According to China's Fire Weather Grades released by Forestry and Grassland Administration in 1995, the assessment indexes of fire weather grades are comprised of the daily highest air temperature $A$, the daily lowest relative humidity $B$, the number of consecutive days without precipitation $C$, the daily maximum wind scale $D$, phenology correction index $E 5$, and partial fire weather indexes. Indexes $A, B, C$, and $D$ are determined using meteorological monitoring parameters. Index $E$ is determined based on the empirical value or monitoring value of phenological changes and substituted into the following equation [24]:

$$
A+B+C+D-E
$$

The standard is used to compute the fire weather grade. Scores are given according to the stepwise intervals. Fire risk grades with major differences are computed based on the meteorological data with minor differences. However, Correction Index $E$ does not consider the soil moisture conditions of forests.

The system computes the fire weather index based on real-time data obtained from the field ecological monitoring station. The computation of fire weather index is smoothed using the logistic regression function [25]. The litter layer soil moisture is introduced for index correction.

\subsection{Smoothing of the Computation of the Fire Weather Index}

According to the industrial standard, the median $X_{i}(i=A, B, C, D)$ scored in stepped intervals of $A, B, C$, and $D$ and the corresponding fire weather index $Y_{i}$ constitute a fitted sample set.

The fitted sample set of Index $A$ is

$\left\{X_{A}, Y_{A}\right\}=\{(2.5,0),(7.5,4),(12.5,8),(17.5,12),(22.5,16),(27.5,20)\}$.

The fitted sample set of Index $B$ is

$\left\{X_{B}, Y_{B}\right\}=\{(75 \%, 0),(65 \%, 4),(55 \%, 8),(45 \%, 12),(35 \%, 16),(25 \%, 20)\}$.

The fitted sample set of Index $C$ is

$\left\{X_{C}, Y_{C}\right\}=\{(0.3,10),(0.9,8),(2.0,6),(3.5,4),(5,2),(7.5,0)\}$.

The fitted sample set of index $D$ is

$\left\{X_{D}, Y_{D}\right\}=\{(0.1,0),(0.9,5),(2.5,10),(6.7,15),(9.4,20),(12.3,25),(15.5,30),(19.0,35),(20.7,40)\}$.

The logistic function

$$
Y_{i}^{\prime}=\frac{K_{i}}{1+e^{\alpha_{i}+\beta_{i} X_{i}}}
$$

is used for fitting the sample. Here, $K_{i}$ is determined by the industrial standard, $K_{i}=\{20,20,10,40\}$, and $\alpha_{i}$ and $\beta_{i}$ are the fitted weights. By fitting based on Equation (2), the computation functions of $A$, $B, C$, and $D$ are

$$
\begin{gathered}
Y_{A}^{\prime}=\frac{20}{1+e^{3.6421-0.2428 X_{A}}} \\
Y_{B}^{\prime}=\frac{20}{1+e^{-6.0701+12.1403 X_{B}}} \\
Y_{C}^{\prime}= \begin{cases}\frac{10}{1+e^{-2.5545+0.8685 X_{C}}}+5 t & \left(X_{C} \leq 10\right) \\
5(t-1) & \left(X_{C}>10\right)\end{cases} \\
Y_{D}^{\prime}=\frac{40}{1+e^{2.2844-0.2396 X_{D}}} .
\end{gathered}
$$


In Equation (5), $t$ is the number of consecutive days with precipitation, and the maximum value $Y_{C}^{\prime}$ is 50. The determination coefficients $R^{2}$ of fitting indexes $A, B, C$, and $D$ in Equation (3)-(6) are $0.9771,0.9771,0.9691$, and 0.9656 , respectively.

\subsection{Computation of Fire Weather Grade}

The phenological correction index $E$ in the industrial standard only considers the degree of coverage. The litter layer soil in forests contains many combustibles. The soil moisture of the litter layer has significant effects on the occurrence and spread of forest fire [26].

In the system, the phenological correction index $E$ is comprised of $Y_{E 1}^{\prime}$ (the degree of coverage) and $Y_{E 2}^{\prime}$ (the litter layer soil moisture index); $Y_{E 1}^{\prime}$ is obtained on the basis of the statistical empirical value, while $Y_{E 1}^{\prime}$ can be increased to 15 in the fire season and to 20 in the core fire season, and can be decreased to 0 beyond the fire season. The litter layer is comprised of a undecomposed layer, a semi-decomposed layer, and a humus layer in a top-down order. The soil moisture sensor can directly measure the volumetric water content of the humus layer and determine the size of $Y_{E 2}^{\prime}$.

There are significant differences in the moisture content of the litter layer soil between different tree species. According to the laboratory test, the volumetric moisture content of the cork oak humus layer ranges between 4.2 and $97.6 \%$ [27]. $Y_{E 2}^{\prime}$ is set to -20 when the volumetric moisture content of the humus layer is above $30 \% . Y_{E 2}^{\prime}$ is set to 0 when the volumetric moisture content of the humus layer is below 5\%. $Y_{E 2}^{\prime}$ and the volumetric moisture content of the humus layer exhibit a linear relationship when the volumetric moisture content of the humus layer is between $5 \%$ and $30 \%$. By linear fitting, we can obtain the computation function of Index $Y_{E 2}^{\prime}$ :

$$
Y_{E 2}^{\prime}=\left\{\begin{array}{cc}
0 & \left(X_{E 2}<5 \%\right) \\
4-80 X_{E 2} & \left(5 \% \leq X_{E 2}<30 \%\right) \\
-20 & \left(X_{E 2} \geq 30 \%\right)
\end{array}\right.
$$

The air temperature, air moisture, precipitation, wind force, and the soil moisture at a depth of $10 \mathrm{~cm}$ obtained from the field ecological monitoring station are substituted into Equations (3)-(7) in sequence to obtain $Y_{i}^{\prime}$. The corrected index of fire weather grade is

$$
Y_{A}^{\prime}+Y_{B}^{\prime}+Y_{C}^{\prime}+Y_{D}^{\prime}+Y_{E 1}^{\prime}+Y_{E 2}^{\prime}
$$

In the system, the fire weather grade is determined by Equation (8). The correspondence relationship is shown in Table 2 [24].

Table 2. Forest fire weather grade form.

\begin{tabular}{cc}
\hline \multicolumn{2}{c}{ Fire Weather Grade } \\
\hline Grade I & $\leq 25$ \\
Grade II & $26 \sim 50$ \\
Grade III & $51 \sim 72$ \\
Grade IV & $73 \sim 90$ \\
Grade V & $\geq 91$ \\
\hline
\end{tabular}

Figure 8 presents the grade of fire weather in the forest microclimate displayed on the PC website in real time, which is updated at an interval of $10 \mathrm{~min}$ in the form of a dashboard. 


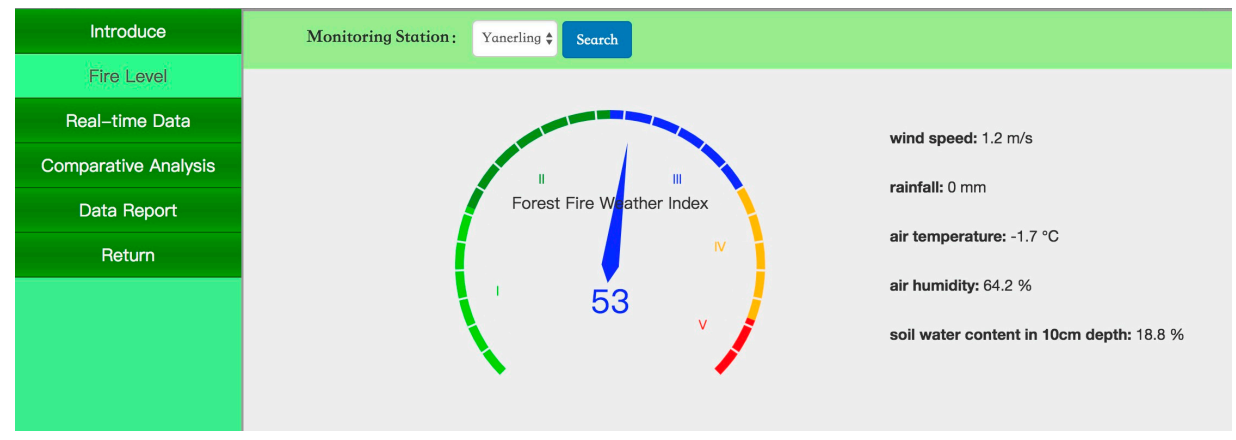

Figure 8. Local forest fire weather grade under the forest microclimate.

\section{Test and Analysis of the Microenvironment Monitoring System for Forest Ecology}

\subsection{Testing of System Stability}

To verify the stability of the system operation, the data of 92 consecutive days obtained from the four field ecological monitoring stations located in Beijing Jiufeng National Forest Park from 1 March 2017 to 31 May 2017 and two monitoring stations located in Inner Mongolia from 31 March 2018 to 30 June 2018. The 13 sensors of 11 types include atmosphere sensors (air temperature, air moisture, carbon dioxide concentration, precipitation, wind speed, and wind direction), light sensors (sunshine hours, total radiation, and light intensity), and soil sensors (two-layer soil moisture and two-layer soil temperature). A network is constructed to monitor the forest ecology. The monitoring data is transmitted in frame via the Beidou satellite or GPRS with each frame containing 72 bytes of data.

Table 3 presents the result of data communication at the field ecological monitoring station in forests. A total of 13,247 frames of field ecological data were acquired with six field ecological monitoring stations in 92 days. The cloud server correctly received 13,058, 13,172, 13,194, and 13,094 frames of ecological data from Yan'er Ridge, Wangjing Tower, Luobadi tower, and Zhai'er Yu, respectively, with correct rates of $98.57 \%, 99.43 \%, 99.59 \%$, and $98.85 \%$ respectively. The cloud server correctly received 13,232 and 13,235 frames of ecological data from Hulun Buir and Zhalantun in Inner Mongolia by GPRS, respectively, with correct rates of $99.89 \%$ and $99.90 \%$, respectively. No data loss lasting for more than $30 \mathrm{~min}$ and data errors occurred. The correct rate of data transmission is high enough to meet the data communication requirements of the microenvironment monitoring system for forest ecology.

Table 3. The communication result of forest microclimate station.

\begin{tabular}{ccccc}
\hline Location & $\begin{array}{c}\text { Communication } \\
\text { Mode }\end{array}$ & $\begin{array}{c}\text { Quantity of Data } \\
\text { Acquired /Frame }\end{array}$ & $\begin{array}{c}\text { Quantity of data Correctly } \\
\text { Transmitted/Frame }\end{array}$ & Correct Rate /\% \\
\hline Yan'er Ridge & Beidou & 13,247 & 13,058 & 98.57 \\
Wangjing & Beidou & 13,247 & 13,172 & 99.43 \\
Tower & Beidou & 13,247 & 13,194 & 99.59 \\
Luobadi & Beidou & 13,247 & 13,094 & 98.85 \\
Zhai'er Yu & GPRS & 13,247 & 13,232 & 99.89 \\
Hulun Buir & GPRS & 13,247 & 13,235 & 99.90 \\
Zhalantun & & & \\
\hline
\end{tabular}

\subsection{Data Analysis}

Yan'er Ridge and Hulun Buir are two typical sites for the field microclimate monitoring and forest fire warning. The mobile phone signal is unavailable in the Yan'er Ridge site, so Beidou satellite is chosen for the data communication. The mobile phone signal is good in the Hulun Buir site, so GPRS is chosen for data communication. The result of these two sites is given to show the validation of the system function. 
The data statements of consecutive days from 1 March 2017 to 31 May 2017 in Yan'er Ridge were downloaded from the cloud server. The variation curves for wind speed, air moisture, air temperature, soil moisture at depths of 10 and $40 \mathrm{~cm}$, and precipitation related to forest fire protection are shown in Figures 9-13. The grades of fire weather in the Yan'er Ridge region from 1 March 2017 to 31 May 2017 computed by the system are shown in Figure 14.

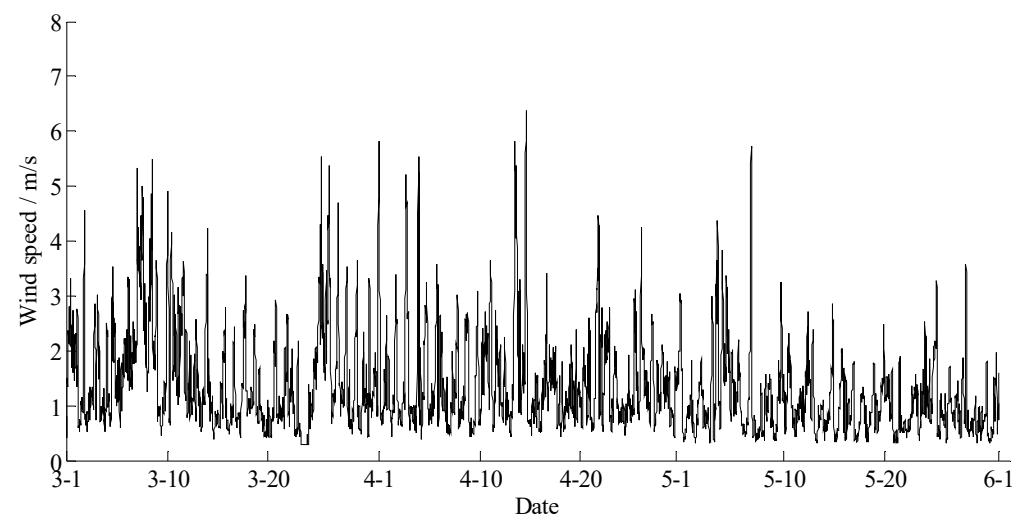

Figure 9. The curve of wind speed in Yan'er Ridge.

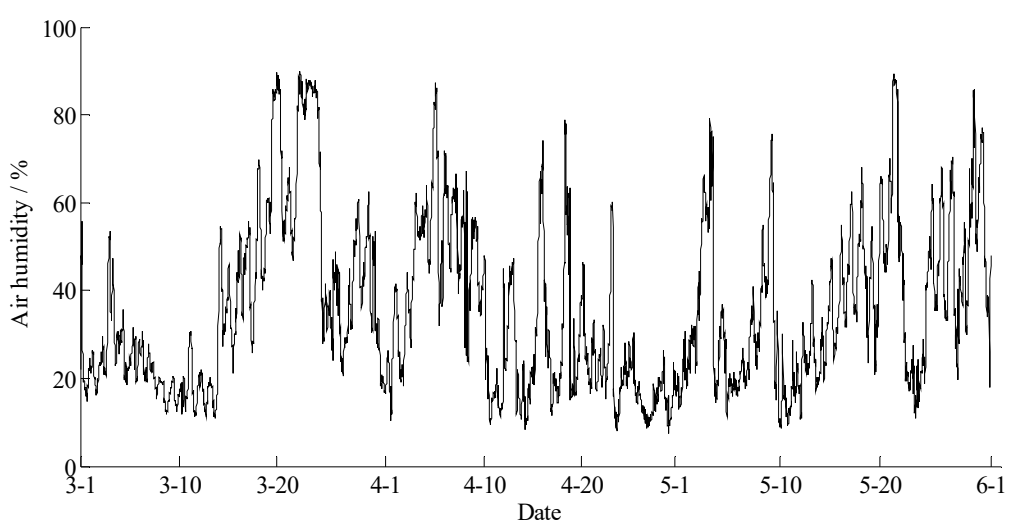

Figure 10. The curve of air humidity in Yan'er Ridge.

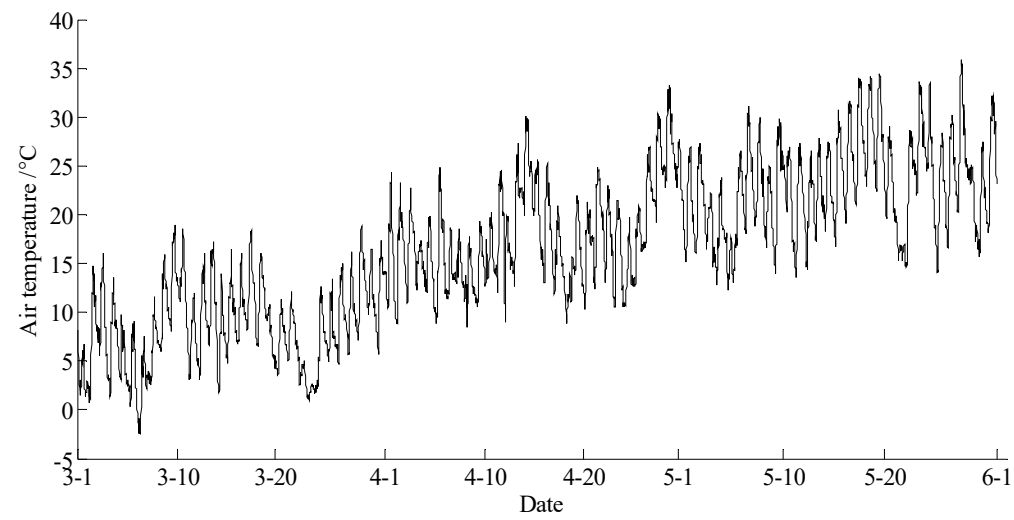

Figure 11. The curve of air temperature in Yan'er Ridge. 


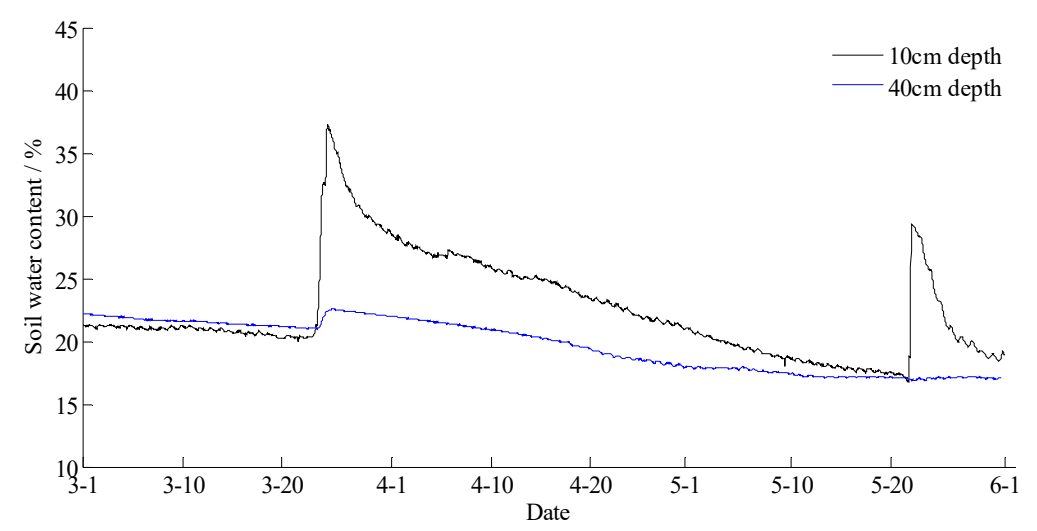

Figure 12. The curves of soil water content in 10 and $40 \mathrm{~cm}$ depth in Yan'er Ridge.

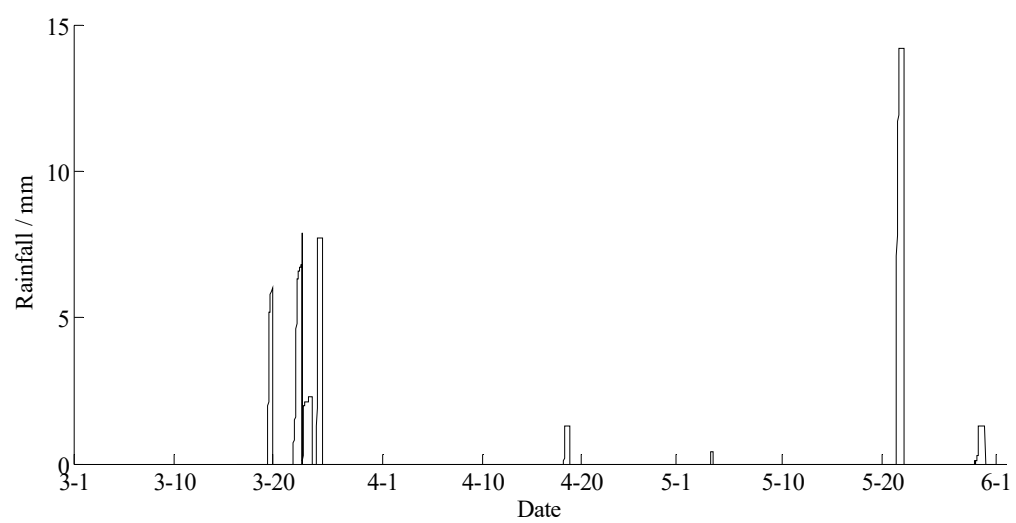

Figure 13. The curve of rainfall in Yan'er Ridge.

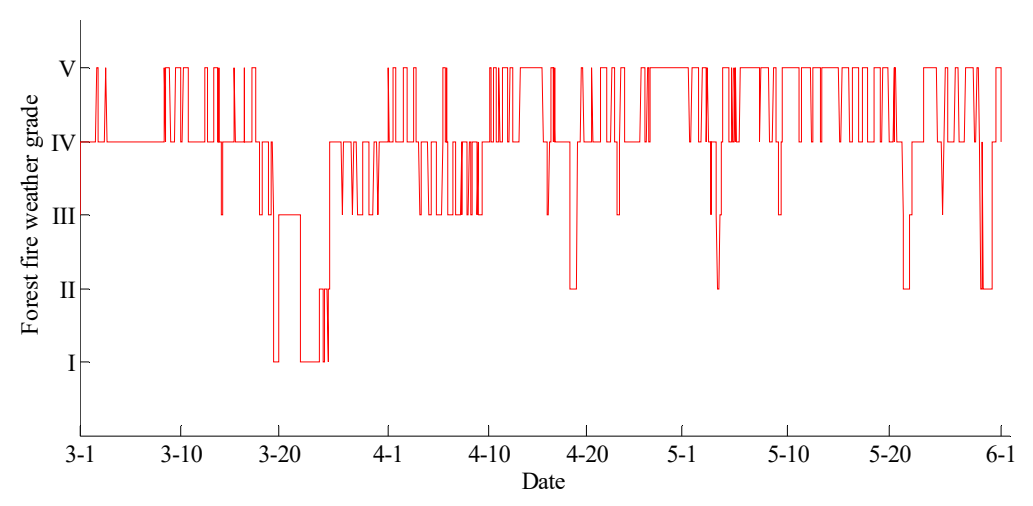

Figure 14. The curve of the forest fire weather grade in Yan'er Ridge.

As shown in Figure 13, continuous precipitation occurred on March 20, 23, and 24. As shown in Figure 14, the moisture delay of soil at depths of 10 and $40 \mathrm{~cm}$ rose rapidly. The index of forest fire protection decreased; the soil moisture decreased slowly after the precipitation stopped. When the moderate rain lasted for a brief period on May 22, the moisture of the soil at a depth of $10 \mathrm{~cm}$ increased, and the fire protection index decreased. The moisture of the soil at a depth of $40 \mathrm{~cm}$ remained unchanged as the sensor was deeply buried and hardly affected by the short-term precipitation that occurred on May 22. The moisture of the soil at depths of 10 and $40 \mathrm{~cm}$ remained unchanged on April 19 and May 30 due to little precipitation.

Based on a comparison between the grade of fire weather in the Yan'er Ridge region in Figure 15 and the variation of precipitation in Figure 14, the fire weather was Grade IV or Grade V in the case of no precipitation in the whole fire protection period, suggesting higher risks of forest fire occurrence and spread. The fire weather was Grade II on April 19, May 22, and May 30 when precipitation lasted 
for a brief period. From March 20 to 24, when precipitation lasted continuously, the fire weather was Grade I or Grade II, suggesting lower risks of forest fire occurrence and spread and effectively forecasting the fire weather grade in this region.

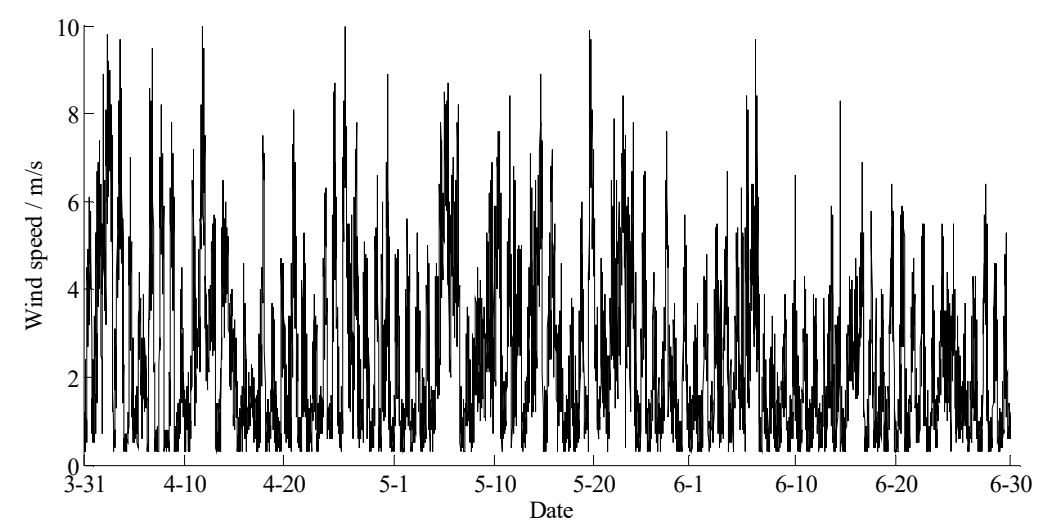

Figure 15. The curve of the wind speed in Hulun Buir.

The data statements of consecutive days from 31 March 2018 to 30 June 2018 in Hulun Buir were downloaded from the cloud server. The variation curves for wind speed, air moisture, air temperature, soil moisture at depths of 10 and $40 \mathrm{~cm}$, and precipitation related to forest fire protection are shown in Figures 15-19. The grades of fire weather in the Hulun Buir computed by the system are shown in Figure 20.

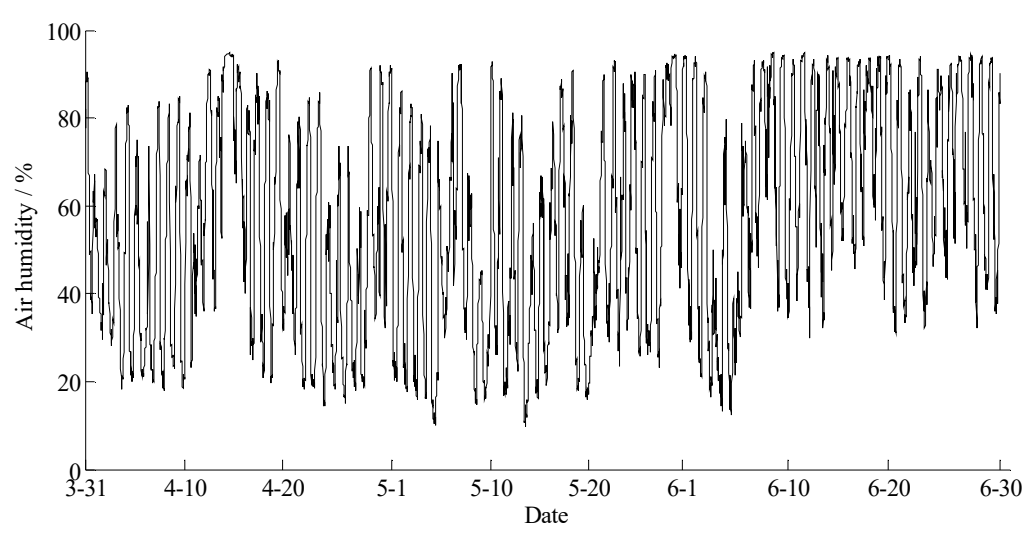

Figure 16. The curve of the air humidity in Hulun Buir.

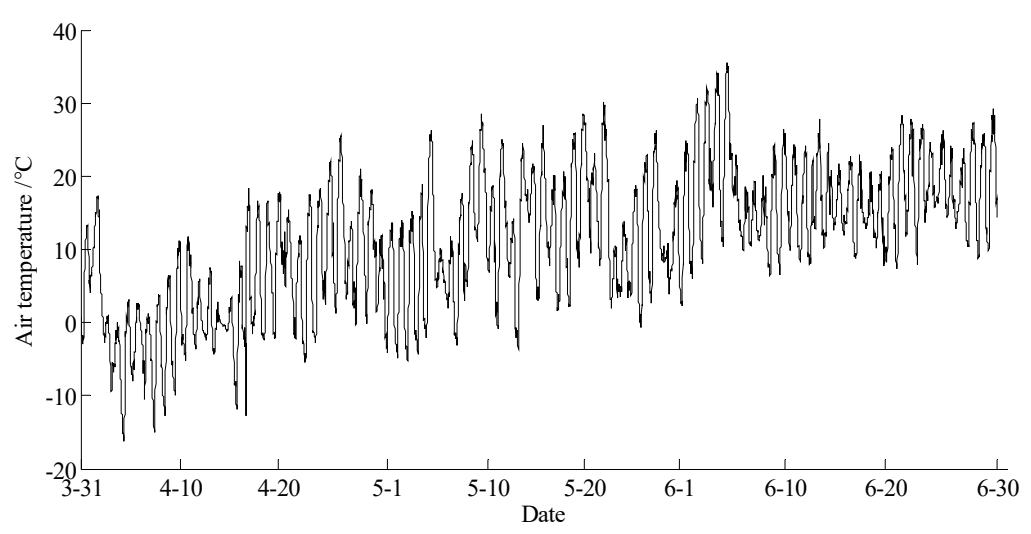

Figure 17. The curve of the air temperature in Hulun Buir. 


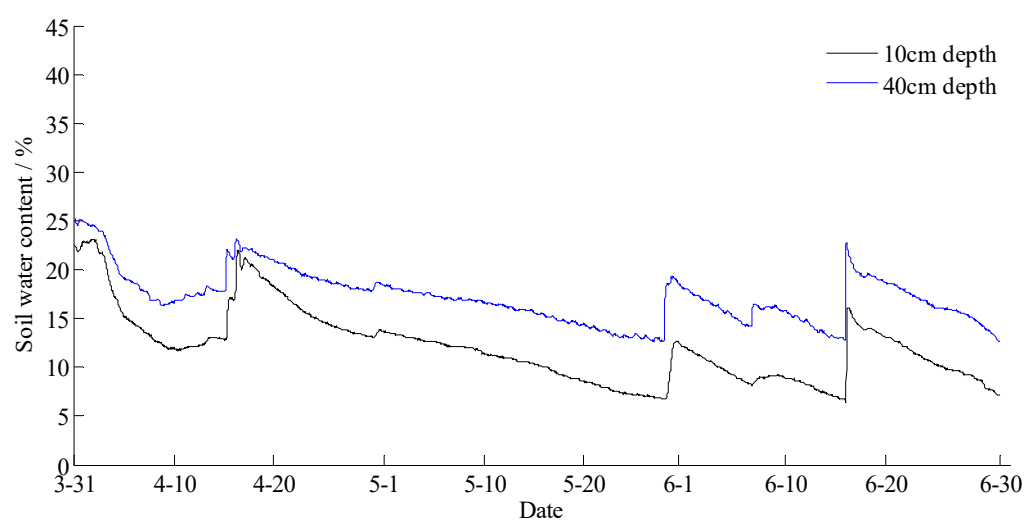

Figure 18. The curves of soil water content at 10 and $40 \mathrm{~cm}$ depths in Hulun Buir.

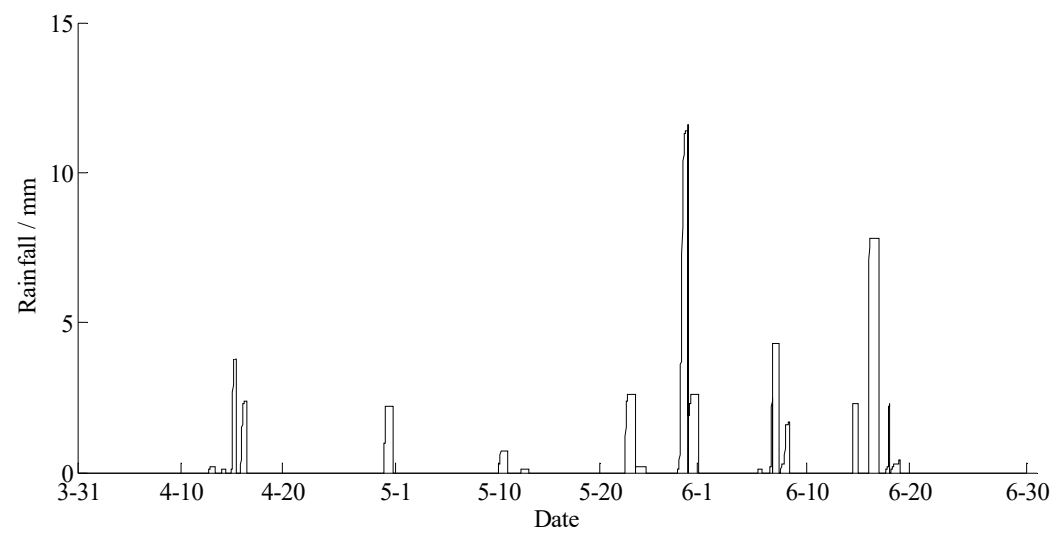

Figure 19. The curve of rainfall in Hulun Buir.

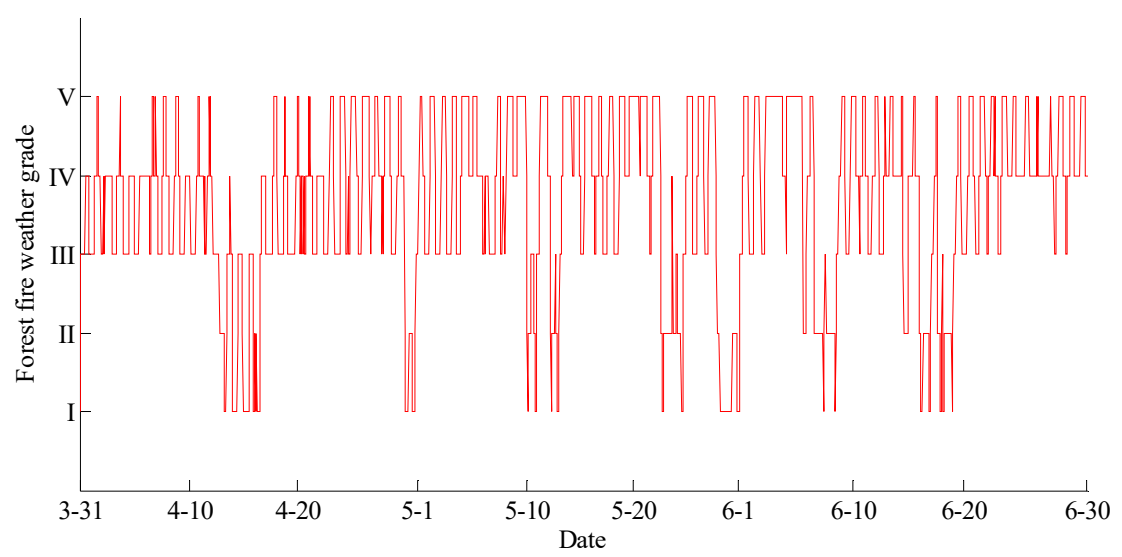

Figure 20. The curve of the forest fire weather grade in Hulun Buir.

As shown in Figure 19, continuous precipitation occurred on April 15, June 6, and June 16. In Figure 18, the moisture delay of soil at depths of 10 and $40 \mathrm{~cm}$ rose rapidly; the index of forest fire protection was decreasing; the soil moisture decreased slowly after the precipitation stopped. Based on a comparison between the grade of fire weather in the Hulun Buir in Figure 20 and the variation of precipitation in Figure 19, the fire weather was Grade III, IV, or V in the case of no precipitation, suggesting higher risks of forest fire occurrence and spread. The fire weather was Grade I or II when precipitation lasted for a brief period, suggesting lower risks of forest fire occurrence and spread and effectively forecasting the fire weather grade in this region. 


\section{Conclusion}

In this paper, we presented the design of a field monitoring network system for forest ecology based on the communication functionality of the Beidou satellite and GPRS. This design achieves real-time monitoring, cloud storage, dynamic query, statement generation, and big data analysis of parameters such as atmosphere, soil, light, and plants. The system solves the step-problem in quantitative scoring of the interval of fire weather indexes using the logistic regression function and achieves a fine forecasting of fire weather grades by introducing the factor of litter layer soil moisture. During the experiment conducted at the Beijing Jiufeng National Forest Park and Inner Mongolia, the system could stably and reliably obtain 11 types of microclimate data of forest with a correct rate of transmission of over $98.57 \%$ by the Beidou satellite and over $99.89 \%$ by GPRS. The system can be used for the big data monitoring of field ecology, an assessment of ecological restoration, meteorological disaster monitoring, accurate irrigation, etc.

Author Contributions: Conceptualization, Y.Z. (Yili Zheng) and Y.Z. (Yandong Zhao); Methodology, Y.Z. (Yili Zheng) and Y.Z. (Yandong Zhao); Software, S.L.; Validation, S.L.; Investigation, W.L.; Resources, W.L.; Writing-Original Draft Preparation, Y.Z. (Yili Zheng); Writing-Review \& Editing, Y.Z. (Yili Zheng) and R.Y.; Visualization, Y.Z. (Yili Zheng); Supervision, Y.Z. (Yandong Zhao); Project Administration, Y.Z. (Yili Zheng) and Y.Z. (Yandong Zhao); Funding Acquisition, Y.Z. (Yili Zheng) and Y.Z. (Yandong Zhao).

Funding: This paper was supported by the Fundamental Research Funds for the Central Universities (No. 2015ZCQ-GX-04), the National Key R\&D Program of China (2017YFD0600901), and the National Natural Science Foundation of China (Grant No. 31670719).

Conflicts of Interest: The authors declare no conflict of interest.

\section{References}

1. Li, S. China Smart Forestry: Top-Level Design and Local Practice; China Forestry Publishing House: Beijing, China, 2016; pp. 3-16.

2. Zhao, F.; Shu, L. Forest Fire Weather and Forecasting and Early Warning; China Forestry Publishing House: Beijing, China, 2014; pp. 101-108.

3. Guo, H.; Yu, W. Study weather grade prediction model of forest-fire risk in Hunan province. J. Central South Univ. For. Technol. 2016, 36, 44-47.

4. Deng, G. Effect of Meteorological Factors on Forest Diseases and Insect Pests in Heilongjiang Province. Ph.D. Thesis, Northeast Forestry University, Harbin, China, 2012.

5. Navarro, G.; Caballero, I.; Silva, G.; Parra, P.C.; Vázquez, Á.; Caldeira, R. Evaluation of forest fire on Madeira Island using Sentinel-2A MSI imagery. Int. J. Appl. Earth Observ. Geoinf. 2017, 58, 97-106. [CrossRef]

6. Natural Resources Canada. Remote Sensing in Forestry. Available online: https://www.nrcan.gc.ca/forests / measuring-reporting/remote-sensing/13429 (accessed on 4 December 2018).

7. Baghdad, N.; Zribi, M. Land Surface Remote Sensing in Agriculture and Forest, 5th ed.; ISTE Press-Elsevier: London, UK, 2016; pp. 363-416.

8. Khamukhin, A.A.; Demin, A.Y.; Sonkin, D.M.; Bertoldo, S.; Perona, G.; Kretova, V. An algorithm of the wildfire classification by its acoustic emission spectrum using Wireless Sensor Networks. J. Phys. Conf. Ser. 2017, 803, 1-7. [CrossRef]

9. Aslan, Y.; Korpeoglu, I.; Ulusoy, Ö. A framework for use of wireless sensor networks in forest fire detection and monitoring. Comput. Environ. Urban Syst. 2012, 36, 614-625. [CrossRef]

10. Son, B.; Her, Y.; Kim, J. A design and implementation of forest-fires surveillance system based on wireless sensor networks for South Korea mountains. Int. J. Comput. Sci. Netw. Secur. 2006, 6, 124-130.

11. Lloret, J.; Garcia, M.; Bri, D.; Sendra, S. A wireless sensor network deployment for rural and forest fire detection and verification. Sensors 2009, 9, 8722-8747. [CrossRef] [PubMed]

12. Hefeeda, M.; Bagheri, M. Wireless Sensor Networks for Early Detection of Forest Fires. In Proceedings of the 2007 IEEE International Conference on Mobile Adhoc and Sensor Systems, Pisa, Italy, 8-11 October 2007; pp. $1-6$.

13. Seo, J.; Park, H. Forest Environment Monitoring Application of Intelligence Embedded based on Wireless Sensor Networks. KSII Trans. Internet Inf. Syst. 2016, 10, 1555-1570. 
14. Lindsey, R.; John, L.; Randall, K.; Mary, B.; Thomas, S.; Rich, H.; David, H.; John, M.; Stephen, S.; Mary, M. Environmental Sensor Applications at USDA Forest Service Experimental Forests: The Smart Forest Network. In Proceedings of the ASA, CSSA, \& SSSA International Annual Meeting, Long Beach, CA, USA, 2-5 November 2014.

15. Ding, F. Construction of Standard System for Localization of Forest Ecosystem. Ph.D. Thesis, Chinese Academy of Forestry, Beijing, China, 2011.

16. Liu, W.; Gao, Z.; Liu, S.; Tao, X.; Zhao, Y. Remote monitoring network for soil moisture based on Iridium communication technology. Trans. Chin. Soc. Agric. Mach. 2015, 46, 316-322.

17. Losso, A.; Corgnati, L.; Bertoldo, S.; Allegretti, M.; Notarpietro, R.; Perona, G. SIRIO: An integrated forest fire monitoring, detection and decision support system-Performance and results of the installation in Sanremo (Italy). Bull. Geor. Acad. Sci. 2012, 170, 530-532.

18. Krstini, D.; Darko, S.; Toni, J. Histogram-Based Smoke Segmentation in Forest Fire Detection System. Inf. Technol. Control 2009, 38, 237-244.

19. Ko, B.; Cheong, K.; Nam, J. Fire detection based on vision sensor and support vector machines. Fire Saf. J. 2009, 44, 322-329. [CrossRef]

20. Li, M.; Qu, L.; Zhao, Q.; Guo, J.; Su, X.; Li, X. Precise point positioning with the Beidou navigation satellite system. Sensors 2014, 14, 927-943. [CrossRef] [PubMed]

21. Mingming, S.; Fei, S.; Aiguo, X. Management system for mechanized straw returning based on BDS. Trans. Chin. Soc. Agric. Mach. 2016, 47, 23-28.

22. Chen, H.; Guo, X.; Wang, F.; Lu, H. Fishery harvesting information compressing and transmitting method based on Beidou short message. Trans. CSAE 2015, 31, 155-160.

23. Liu, X. Establishment of Forest Environment Factor Monitoring Data Management System. Master's Thesis, Beijing Forestry University, Beijing, China, 2015.

24. China National Forestry Administration. National Forest Fire Weather Ranks; China Standard Press: Beijing, China, 1995; pp. 1-8.

25. Zhang, W.; Wang, F.; Guo, Y.; Zheng, Y. Weather model level of forest fire danger based on logistic regression. J. Northeast For. Univ. 2013, 41, 121-122.

26. Chaparro, D.; Vall-Llossera, M.; Piles, M.; Camps, A.; Rüdiger, C. Low soil moisture and high temperatures as indicators for forest fire occurrence and extent across the Iberian Peninsula. In Proceedings of the 2015 IEEE International Geoscience and Remote Sensing Symposium (IGARSS), Milan, Italy, 26-31 July 2015; pp. 3325-3328.

27. Yan, X.; Zheng, X.; Zhao, Y.; Zhao, Y. Measurement method of forest floor and soil water content based on standing wave ratio. Trans. Chin. Soc. Agric. Mach. 2017, 48, 278-283. 